\title{
Diabetes Mellitus Associado à Mutação Mitocondrial A3243G: Freqüência e Caracterização Clínica
}

\begin{abstract}
RESUMO
Diabetes mitocondrial é freqüentemente associado à mutação mitocondrial A3243G. A prevalência desse subtipo de diabetes na população diabética varia de 0,5 a $3 \%$, dependendo do grupo populacional estudado. Objetivo: Examinar a freqüência e o quadro clínico do diabetes associado com a mutação mitocondrial A3243G em pacientes brasileiros com tolerância a glicose alterada. Métodos: A população estudada foi composta por 78 indivíduos portadores de diabetes mellitus tipo 1 (grupo I), 148 diabéticos tipo 2 (grupo II), 15 diabéticos tipo 1 ou tipo 2 portadores de disacusia (grupo III) e 492 indivíduos da comunidade nipo-brasileira com vários graus de intolerância a glicose. O DNA foi extraído de leucócitos do sangue periférico e a mutação A3243G foi determinada através da amplificação por PCR e digestão por Apa 1. Em alguns pacientes, o DNA também foi extraído da mucosa oral e folículo capilar. A mutação A3243G foi identificada em três indivíduos, todos do grupo III, resultando em uma prevalência de $0,4 \%$. Os carreadores da mutação apresentavam diagnóstico do diabetes em idade jovem, índice de massa corpórea normal ou baixo e requerimento de insulina. Conclusão: Diabetes mitocondrial é um subtipo raro de diabetes em nossa população e deve ser investigado naqueles indivíduos portadores de diabetes e surdez. (Arq Bras Endocrinol Metab 2007;51/4:559-565)
\end{abstract}

Descritores: Diabetes mellitus; Diabetes miticondrial; Surdez; Mutação A3243G

\section{ABSTRACT}

Diabetes Mellitus Associated With the Mitochondrial Mutation A3243G: Frequency and Clinical Presentation.

Maternal inherited diabetes and deafness (MIDD) has been related to an $A$ to G transition in the mitochondrial RNA Leu (UUR) at base pair 3243. The prevalence of MIDD in the diabetes population ranges between $0.5-3.0 \%$ depending on the ethnic background. Aim: To examine the frequency and clinical features of diabetes associated with this mutation in Brazilian patients with glucose intolerance. Methods: The study population comprised: 78 type 1 diabetic subjects (group I), 148 patients with type 2 diabetes (group II), 15 patients with either type 1 or type 2 diabetes and hearing loss (group III) and 492 Japanese Brazilians with varying degrees of glucose intolerance. DNA was extracted from peripheral blood leucocytes and the A3243G mutation was determined by PCR amplification and Apa 1 digestion. In some individuals DNA was also extracted from buccal mucosa and hair follicles. The 3243 bp mutation was found in three individuals, all from group III, resulting in a prevalence of $0.4 \%$. These subjects had an early age of diagnosis of diabetes, low or normal body mass index and requirement of insulin therapy. In conclusion MIDD is rare in our population and should be investigate in patients with diabetes and deafness. (Arq Bras Endocrinol Metab 2007;51/4:559-565)

Keywords: Diabetes mellitus; Mitochondrial diabetes; Deafness; Mutation A3243G artigo original

\author{
JoÃo EDUARDO N. SALLES \\ LARISSA BRESGUNOV KALININ \\ SANDRA ROBERTA G. FERREIRA \\ TERESA KASAMATSU \\ REGINA S. MOISÉS
}

Disciplina de Endocrinologia, Departamento de Medicina (JENS, LBK, TK \& RSM) e Departamento de Medicina Preventiva (SRGF), Universidade Federal de São Paulo, SP.

Recebido em 21/07/06

Revisado em 04/12/06

Aceito em 13/12/06 
A LTERAÇÕES NO DNA mitocondrial (DNA mit) têm sido implicadas com o desenvolvimento de diabetes mellitus (DM), sendo a substituição de A por $G$ na posição 3243 no gene do RNAt da leucina a mutação mais comumente associada (1). A segregação dessa mutação com diabetes foi inicialmente reportada por van den Ouweland e col. em 1992, em uma grande família holandesa (2), sendo posteriormente proposto o nome de MIDD (Maternal Inherited Diabetes and Deafness) para esse subtipo de diabetes (3). Nesses pacientes, o DM pode apresentar-se como não dependente de insulina com posterior necessidade de insulinoterapia ou como dependente de insulina desde o início (4). A suspeita clínica é feita pela presença de perda auditiva neurosensorial, transmissão materna do diabetes, índice de massa corporal normal ou baixo e baixa estatura.

Estudos têm sido realizados em diferentes grupos de diabéticos para investigar-se a prevalência de MIDD. Na população diabética em geral, a freqüência reportada é de 0,5 a 2,6\%, dependendo dos critérios de seleção (5-7). Entre os japoneses parece haver uma maior prevalência, sendo reportado corresponder entre 1 a 3\% de todos os casos de diabetes no Japão (8-10). Em nosso meio não há dados da prevalência da mutação A3243G entre os portadores de anormalidades no metabolismo dos carboidratos.

Os nipo-brasileiros apresentam uma alta prevalência de anormalidades no metabolismo dos carboidratos, sendo que a prevalência de diabetes mellitus é uma das mais altas em todo o mundo (11).

No presente estudo, temos por objetivo determinar a freqüência da mutação A3243G no DNA mitocondrial em diferentes grupos de portadores de anormalidades no metabolismo dos carboidratos e as características clínicas desse subtipo de diabetes.

\section{PACIENTES E MÉTODOS}

\section{População estudada}

A população estudada consistiu de 733 indivíduos, não relacionados, portadores de intolerância a glicose que foram divididos em quatro grupos:

Grupo I: 78 indivíduos portadores de DM tipo 1, sendo 44 do sexo masculino e 34 do sexo feminino, com idade média de $23,3 \pm 5,8$ anos. Foram considerados portadores de diabetes mellitus tipo 1 aqueles indivíduos nos quais o diagnóstico foi feito antes dos 30 anos de idade, com início agudo dos sintomas e em insulinoterapia desde o diagnóstico. Não foram utilizados critérios imunológicos para definição do diabetes mellitus tipo 1 .

Grupo II: 148 indivíduos portadores de DM tipo 2, sendo 43 do sexo masculino e 105 do sexo feminino com idade média de 58,5 $\pm 10,4$ anos. Destes, 71 apresentavam antecedente materno de diabetes. Foram considerados portadores de diabetes mellitus tipo 2 aqueles nos quais o diagnóstico foi feito com idade igual ou superior a 30 anos, que permaneceram por pelo menos um ano após o diagnóstico sem a necessidade de insulinoterapia para o controle metabólico e não apresentavam evidência prévia de cetoacidose.

Grupo III: 15 indivíduos portadores de DM (classificado como tipo 1 ou tipo 2) associado com disacusia, sendo 5 do sexo masculino e 10 do sexo feminino, com idade média de 35,6 $\pm 18,2$ anos.

Grupo IV: 492 indivíduos da comunidade nipobrasileira de Bauru, sendo 226 do sexo masculino e 266 do sexo feminino, com idade média de 59,0 $\pm 10,9$ anos. Destes, 257 eram portadores de diabetes mellitus, 140 de tolerância a glicose diminuída e 95 glicemia de jejum alterada. Esses indivíduos fazem parte do Japanese Brazilian Diabetes Study Group, um estudo populacional com o objetivo de se investigar a ocorrência de intolerância a glicose numa comunidade urbana de primeira e segunda geração de nipobrasileiros vivendo na cidade de Bauru, SP. Os critérios de seleção e recrutamento desses indivíduos foram previamente descritos (11).

O grau de tolerância a glicose foi determinado através de teste oral de tolerância a glicose, exceto nos indivíduos com DM previamente diagnosticado, e interpretado de acordo com os critérios da OMS (12). Exame audiométrico foi realizado por fonoaudióloga, que examinou o limiar tonal e índice porcentual de reconhecimento de fala nos indivíduos carreadores da mutação A3243G. Pesquisa de distrofia macular foi realizada por oftalmologista experiente de acordo com os critérios reportados por Massin e col (13). Função da célula $\beta$ pancreática foi avaliada através dos níveis de peptídeo $\mathrm{C}$ em jejum nos carreadores da mutação A3243G. Pacientes com valores inferiores a $0,51 \mathrm{ng} / \mathrm{ml}$ foram considerados insulino-deficientes (14). Peptídeo C foi medido através de ensaio imunofluorimétrico em fase sólida (Wallac Auto Delfia C-peptide kit). As características clínicas da população estudada encontram-se na tabela 1.

O protocolo de estudo foi aprovado pelo Comitê de Ética em Pesquisa da Universidade Federal de São Paulo e os participantes foram informados a respeito do estudo e assinaram previamente um termo de consentimento.

\section{Detecção da mutação A3243G}

DNA total foi extraído de leucócitos de sangue periférico utilizando um kit comercial (Puregene DNA Isolation Kit, Gentra System, Minneapolis, MN, USA). Em alguns indivíduos, o DNA também foi extraído de mucosa oral e folículo capilar. Amplificou-se a região de interesse através de reação de PCR utilizando-se os seguintes iniciadores: 5' CGTTTGTTC AACGATTAAAG-3' e 5'-CACGTTGG GGCCTTTG CGTA-3' (10). O fragmento gerado de 422 pares de bases foi, então, submetido a digestão com a enzima de restrição Apa I (Gibco BRL, USA), por 1 hora a $37^{\circ} \mathrm{C}$. As amostras foram, então, submetidas a eletroforese 
Tabela 1. Características clínicas da população estudada de acordo com os grupos: grupo I (portadores de DM tipo 1), grupo II (portadores de DM tipo 2), grupo III (portadores de DM e disacusia), grupo IV (indivíduos nipo-brasileiros com intolerância à glicose).

\begin{tabular}{|c|c|c|c|c|}
\hline & $\begin{array}{l}\text { Grupo I } \\
(n=78)\end{array}$ & $\begin{array}{l}\text { Grupo II } \\
(n=148)\end{array}$ & $\begin{array}{c}\text { Grupo III } \\
(n=15)\end{array}$ & $\begin{array}{r}\text { Grupo IV } \\
(n=492)\end{array}$ \\
\hline Sexo (M/F) & $44 / 34$ & $43 / 105$ & $5 / 10$ & $226 / 266$ \\
\hline Idade & $23,3 \pm 5,8$ & $58,5 \pm 10,4$ & $35,6 \pm 18,2$ & $59 \pm 10,9$ \\
\hline IMC $\left(\mathrm{kg} / \mathrm{m}^{2}\right)$ & $22,7 \pm 2,8$ & $28,7 \pm 4,6$ & $20,2 \pm 04$ & $25,2 \pm 3,7$ \\
\hline $\begin{array}{l}\text { Idade ao diagnóstico } \\
\text { da intolerância a glicose } \\
\text { (anos) }\end{array}$ & $12,3 \pm 4$ & $49,2 \pm 10,5$ & $21,6 \pm 17,7$ & $60 \pm 11$ \\
\hline
\end{tabular}

Dados expressos em média \pm desvio-padrão.

em gel de agarose a $2 \%$, tratadas com brometo de etídio e visualizados sob luz ultravioleta. A presença da mutação gera um sítio de restrição para a enzima Apa I, resultando em fragmentos adicionais de $210 \mathrm{pb}$ e $212 \mathrm{pb}$. Quando submetidos a eletroforese em gel de agarose ambos os fragmentos de $210 \mathrm{pb}$ e $212 \mathrm{pb}$ aparecem como uma única banda, levando com isso a uma melhor sensibilidade na análise por PCR-RFLP. A porcentagem de fragmentos clivados foi determinada através de análise densitométrica.

\section{Análise estatística}

Os dados são apresentados como media \pm desvio-padrão. A correlação entre idade e heteroplasmia foi calculada por coeficiente de correlação linear de Pearson. $\mathrm{P}<0,05$ foi considerado estatisticamente significante.

\section{RESULTADOS}

A mutação A3243G foi identificada em apenas três indivíduos, todos pertencentes ao grupo III. Os três pacientes afetados apresentavam idade precoce ao diagnóstico do diabetes mellitus, eram não obesos e insulino-deficientes de acordo com os níveis basais de peptídeo C. As características clínicas desses indivíduos são apresentadas na tabela 2 .

Membros das famílias dos três indivíduos nos quais identificou-se a mutação foram recrutados e, se disponíveis, submetidos a pesquisa da mutação A3243G, teste oral de tolerância a glicose e audiometria. Apresentamos na figura 1 o heredograma das famílias estudadas.

$\mathrm{Na}$ família $\mathrm{Fl}$, além do propósito, não identificamos nenhum outro membro portador da mutação A3243G. Na mãe do probando, portadora de tolerância normal a glicose, a pesquisa foi também realizada em células da mucosa oral e folículo capilar, sendo a mutação indetectável nesses tecidos. Teste de paternidade, utilizando-se marcadores polimórficos no DNA, comprovou a relação familiar informada. $\mathrm{O}$ probando, além do diabetes mellitus e disacusia neuro-sensorial, apresentou Síndrome de Wolff-Parkinson-White, tendo sido submetida à ablação de via acessória lateral esquerda. A apresentação do DM foi em cetoacidose e desde o diagnóstico vinha em uso de insulinoterapia. Durante o período de seguimento, desenvolveu miocardiopatia, conforme reportagem anterior (15), e veio a falecer de edema pulmonar agudo.

Na família F2, a mutação também foi identificada em uma irmã, tia materna e prima do propósito. Todos os carreadores da mutação apresentavam diabetes mellitus, e disacusia foi verificada nos indivíduos nos quais foi possível a realização de audiometria. A mãe do propósito, já falecida por ocasião da investigação, apresentava uma história de disacusia diagnosticada aos 25 anos e diabetes mellitus, aos 35 anos. O diagnóstico de DM no probando foi feito através de exame de rotina. O tratamento foi com sulfoniluréia durante o primeiro ano, porém após esse período necessitou de insulinoterapia para controle metabólico. Como complicações neurológicas apresenta movimentos coreicos, incoordenação motora e disartria.

Na família F3, a mutação também foi identificada na mãe, duas irmãs e duas sobrinhas do propósito. Nessa família, a mutação A3243G não mostrou segregação completa com o diabetes, sendo que apenas o propósito apresentava diabetes mellitus, enquanto os demais membros carreadores da mutação apresentavam tolerância normal a glicose. Disacusia neuro-sensorial foi identificada nos indivíduos F3 II-1 e F3 II-2, enquanto os indivíduos F3 III-1 e F3 III-2 apresentavam teste audiométrico normal. O probando foi tratado com insulina desde o diagnóstico do DM. Nos indivíduos F2 I-3 e F3 I-2, a mutação foi identificada apenas em DNA extraído de folículo capilar e mucosa oral.

Investigamos a correlação entre a porcentagem de heteroplasmia da mutação A3243G em leucócitos e a idade dos indivíduos em que foi feita a análise genética. Verificamos uma correlação negativa entre essas 
Tabela 2. Características dos probandos carreadores da mutação.

\begin{tabular}{lccc}
\hline & Probando F1 & Probando F2 & Probando F3 \\
Sexo & Feminino & Feminino & Masculino \\
Idade (anos) & 38 & 30 & 38 \\
IMC (kg/m²) & 17,1 & 22,3 & 21 \\
Idade ao diagnóstico (anos) & 24 & 25 & 19 \\
Tratamento atual DM & insulina & insulina & insulina \\
História Materna DM & Não & Sim & Não \\
Peptídeo C jejum (ng/ml) & 0,31 & 0,48 & $<0,05$ \\
Audiograma & perda neuro-sensorial & perda neuro-sensorial & perda neuro-sensorial \\
Complicações mitocôndrio-relacionadas & cardiopatia & movimentos coreícos & distúrbios TGl \\
& atrofia cerebral & & \\
\hline
\end{tabular}

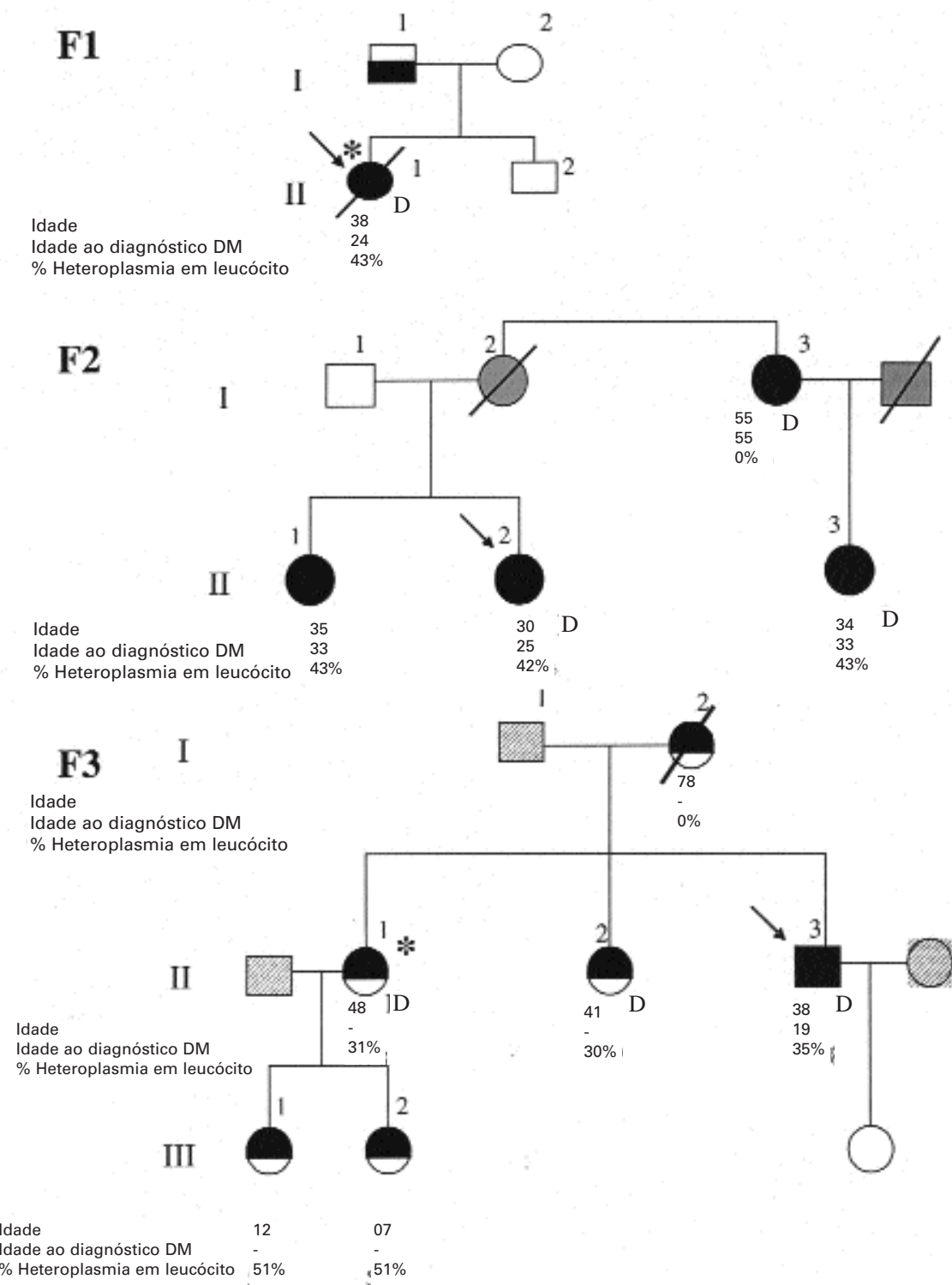

Figura 1. Heredogramas das famílias afetadas. Números romanos à esquerda indicam a geração e números acima dos símbolos indicam os indivíduos de cada geração.

$\square$ TGN; OCarreadores da mutação DM; $\odot$ Carreadores da mutação TGN; $\square$ Portadores de diabetes; $\bigcirc$ Não avaliados. Setas indicam os propósitos. D indica disacusia. 


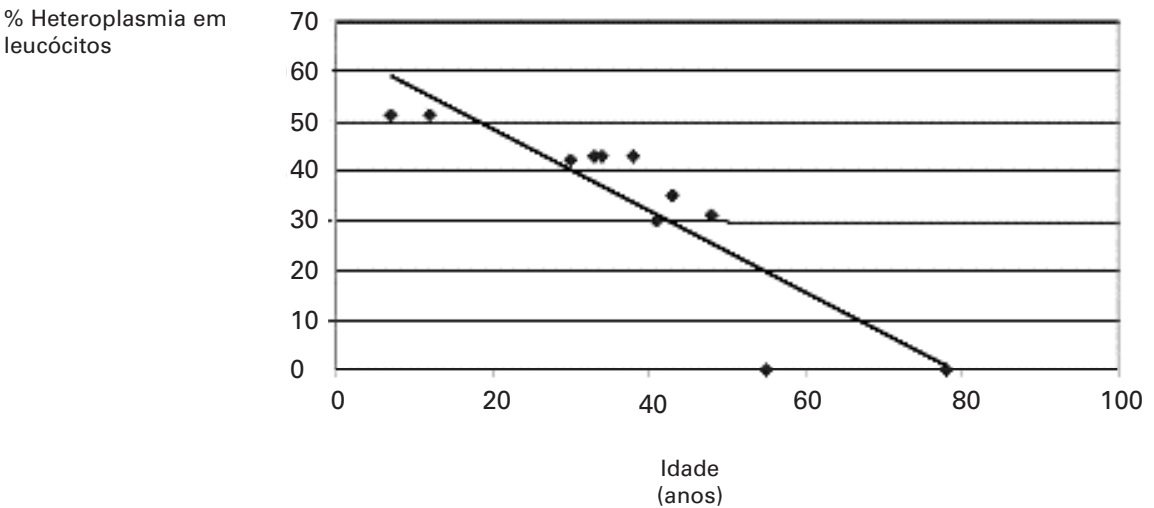

Figura 2. Correlação entre porcentagem de heteroplasmia da mutação $A 3243 G$ em leucócitos e idade no momento do estudo molecular.

duas variáveis, indicando um declínio dependente da idade na porcentagem de heteroplasmia em leucócitos $(\mathrm{r}=-0,8204, \mathrm{p}=0,003)$ (figura 2$)$.

\section{DISCUSSÃO}

MIDD é um subtipo de diabetes que apresenta um amplo espectro clínico, podendo inicialmente ser classificado como DM tipo 1 ou DM tipo 2. Em um grande estudo multicêntrico, verificou-se que $13 \%$ dos pacientes com MIDD eram dependentes de insulina desde o diagnóstico, 46\% necessitaram de insulinoterapia após uso de medicação oral e $41 \%$ eram independentes de insulina (16). Portanto, na população estudada, procuramos cobrir esse espectro selecionando indivíduos clinicamente classificados como portadores de DM tipo 1, DM tipo 2 e DM associado a disacusia. Entre os nipo-brasileiros, uma população com alta prevalência de anormalidades no metabolismo dos carboidratos, consideramos como traço a intolerância a glicose (diabetes mellitus, tolerância a glicose diminuída e glicemia de jejum alterada), uma vez que tolerância a glicose diminuída e glicemia de jejum alterada podem ser precursores do DM tipo 2.

Em indivíduos portadores de defeitos no genoma mitocondrial, as células podem apresentar uma mistura variável de DNA mitocondrial normal e mutado, fenômeno esse denominado de heteroplasmia. Freqüentemente há variações no nível de DNA mitocondrial mutante entre os diferentes tecidos em um mesmo indivíduo $(17,18)$, sendo geralmente níveis mais elevados observados em tecidos pós-mitóticos, tais como músculo esquelético, e menores níveis em tecidos que sofrem rápidas divisões celulares, tais como os leucócitos (19). Entretanto, biópsia muscular é um método invasivo que não pode ser utilizado em avaliação rotineira, e os leucócitos de sangue periférico são fáceis de serem obtidos e, portanto, freqüentemente utilizados para pesquisa de mutações genéticas. No presente estudo, e de forma similar aos estudos previamente publicados, pesquisamos a mutação em DNA mitocondrial extraído de leucócitos, possibilitando com isso o estudo de um número razoável de pacientes. Verificamos um decréscimo na porcentagem de heteroplasmia em leucócito associado ao envelhecimento, dados esses concordantes com estudos anteriores $(20,21)$. Em dois indivíduos a porcentagem de heteroplasmia em leucócitos estava abaixo do limite de detecção do método, sendo a mutação detectada apenas quando pesquisada em DNA extraído de células de mucosa oral ou folículo capilar. Isso implica que, em indivíduos idosos e com forte suspeita clínica de MIDD, se a pesquisa da mutação A3243G for negativa em amostras extraídas de leucócitos, a análise deve ser repetida utilizando-se DNA mit extraído de outros tecidos como, por exemplo, células de mucosa oral ou folículo capilar.

Entre os 733 indivíduos portadores de anormalidades no metabolismo dos carboidratos examinados, a mutação A3243G foi identificada em três indivíduos, resultando em uma prevalência geral de $0,4 \%$. Essa prevalência é similar à encontrada em uma população brasileira do Rio Grande do Sul portadora de diabetes tipo 2 (22), e entre caucasianos europeus $(6,23,24)$, porém inferior ao reportado entre franceses (6) ou japoneses (8-10). Essas variações podem ser explicadas pelas diferenças étnicas entre as populações estudadas e também pelos diferentes critérios de seleção entre os estudos. $\mathrm{Ng}$ e col. identificaram a mutação em 3\% dos portadores de DM tipo 2 com início precoce da doença ( $<40$ anos de idade) e história 
materna de DM, porém em apenas 0,3\% dos indivíduos com início do DM após os 40 anos de idade e sem antecedente familiar para a doença (25). Já t’Hart e col., utilizando um critério mais estrito, ou seja, herança materna do DM e presença de surdez, verificou uma prevalência da mutação A3243G de 10\% entre portadores de DM tipo 2 (26). Em nossa casuística de portadores de DM tipo 2, o antecedente materno de DM foi verificado em 48\% (71 de 148) dos indivíduos, porém em apenas 11\% (16 de 148) o diagnóstico foi feito antes dos 40 anos de idade, talvez sendo essa uma das razões para o não encontro da mutação nesse grupo de indivíduos. Entretanto, quando o critério de seleção foi a presença de DM e disacusia, a mutação foi verificada em 20\% (3 de 15) dos indivíduos. Esses dados são concordantes com estudos anteriores que identificaram a presença da mutação A3243G em 10 a $60 \%$ dos indivíduos portadores de DM e surdez $(26,27)$.

Em relação ao fenótipo, verificamos alguma heterogeneidade entre os dos carreadores da mutação A3243G. Por exemplo, na família F3 a penetrância do diabetes mellitus não foi completa, diferentemente do observado nas outras duas famílias. Os indivíduos F3 II-1 e F3 II-2, com 45 e 41 anos de idade, respectivamente, apresentavam tolerância normal a glicose. Já os indivíduos F3 III-1 e F3 III-2 são mais jovens do que a idade média de diagnóstico do diabetes mellitus e, portanto, podem ainda vir a apresentar a doença. A ocorrência de menores graus de heteroplasmia nas células $\beta$ pancreáticas desses indivíduos pode explicar o não desenvolvimento do diabetes. Entretanto, Ciafaloni e col. reportaram porcentagens similares de genoma mutante em tecidos clinicamente afetados e não afetados em pacientes com MELAS (28).

Nos indivíduos nos quais foi possível a realização de audiometria, verificamos que todos os adultos carreadores da mutação apresentavam disacusia neurosensorial de graus moderado a severo. Esses achados são concordantes com os obtidos por outros autores que verificaram perda auditiva em 93 a 100\% dos pacientes $(7,16)$. Deve-se observar que, em nossa casuística, as crianças e/ou adolescentes afetados não apresentaram nenhuma manifestação clínica tal como diabetes mellitus ou disacusia, sugerindo que a idade tenha um papel patogênico no desenvolvimento dessas condições. Um seguimento prospectivo desses jovens nos permitirá observar se haverá ou não desenvolvimento de diabetes e/ou disacusia.

Quanto ao diabetes mellitus, verificamos as seguintes características: diagnóstico antes dos 35 anos de idade, peso adequado ou baixo e necessidade de insulina na maioria dos casos. Esses resultados confirmam estudos prévios $(1,4,16,29)$.

A transmissão materna da mutação foi confirmada em duas das três famílias. Na família Fl, apenas o probando apresentou a mutação A3243G. Para excluirmos a possibilidade de que sua mãe apresentasse a mutação, porém abaixo do limiar de detecção do método, DNA para estudo molecular foi também extraído de mucosa oral e folículo capilar, tecidos esses que apresentam maior porcentagem de DNA mutante em comparação com leucócitos $(20,21,30)$. A mutação não foi identificada em nenhum desses tecidos maternos, indicando tratar-se do aparecimento da mutação de novo no caso índice. Há, na literatura, apenas três descrições anteriores de mutação A3243G de aparecimento de novo (31-33).

Entre as complicações associadas à mitocôndria, identificamos nos pacientes estudados a presença de cardiomiopatia, Síndrome de Wolff-Parkinson-White e distúrbios neurológicos. A distrofia macular caracterizada por lesões hiperpigmentadas na região macular e do nervo óptico associada com alterações tróficas do epitélio retiniano foi descrita como característica de pacientes com MIDD (13). Guillausseau e col. demonstraram uma alta prevalência $(86 \%)$ dessas lesões em pacientes franceses com MIDD considerando-a patognomônica da doença, podendo, inclusive, ser usada como marcador para selecionar pacientes com possível MIDD (16). Em nossos pacientes com MIDD, a presença de distrofia macular não foi verificada. Similarmente entre os japoneses, Suzuki e col. (20) verificaram uma baixa prevalência dessa complicação, dado esse sugerindo um possível determinante étnico para a presença dessa condição.

Em conclusão, a mutação A3243G é responsável por apenas uma pequena porcentagem de anormalidades no metabolismo dos carboidratos em nosso meio. Entretanto, o encontro de diabetes mellitus, peso adequado ou baixo associado a disacusia deve alertar para a possibilidade de MIDD. Esse subtipo de diabetes requer um seguimento mais de perto pela possibilidade de desenvolvimento de complicações peculiares tais como cardiomiopatia, distúrbios de condução cardíaca e sintomas neurológicos.

\section{AGRADECIMENTOS}

Agradecemos aos membros do Japanese Brazilians Diabetes Study Group pelos dados obtidos entre os nipo-brasileiros. 


\section{REFERÊNCIAS}

1. Maassen JA, Leen M, van Essen E, Heine RJ, Nijpels G, Tafrechi RSJ. Mitochondrial diabetes: mechanism and clinical presentation. Diabetes 2004;53(suppl. 1):S103-9.

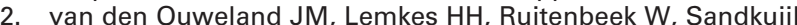
LA, de Vijlder MF, Maassen JA, et al. Mutation in mitochondrial tRNA(Leu)(UUR) gene in a large pedigree with maternally transmitted type II diabetes mellitus and deafness. Nat Genet 1992:5:368-71.

3. van den Ouweland JM, Lemkes $H H$, Trembath RC, Roos R, Velho G, Maassen JA, et al. Maternally inherited diabetes and deafness is a distinct subtype of diabetes and associates with a single point mutation in the mitochondrial tRNA(Leu(UUR)) gene. Diabetes 1994;43(6):746-51.

4. Guillausseau PJ, Dubois-Laforgue D, Massin P, Laloi-Michelin $\mathrm{M}$, Bellanné-Chantelot $\mathrm{C}$, Gin $\mathrm{H}$; on behalf of the GEDIAM Group. Heterogeneity of diabetes phenotype in patients with 3243 bp mutation of mitochondrial DNA (Maternally Inherited Diabetes and Deafness or MIDD). Diabetes Metab 2004;30:181-6.

5. Alcolado JC, Majid A, Brockinton M, Sweeney MG, Morgan R, Ress $A$, et al. Mitochondrial gene defects in patients with NIDDM. Diabetologia 1994;37(4):372-6.

6. Vionnet N, Passa P, Froguel P. Prevalence of mitochondrial gene mutations in families with diabetes mellitus. Lancet 1993:342:1429-30.

7. Lehto $M$, Wipeno $C$, Ivarsson SA, Lindigren $C$, Tuomi T, Groop L, et al. High frequency of mutations in MODY and mitochondrial genes in Scandinavian patients with familial early-onset diabetes. Diabetologia 1999;42(9):1131-7.

8. Otabe S, Sakura H, Shimokawa K, Mori Y, Kadowaki H, Yasuda $\mathrm{K}$, et al. The high prevalence of the diabetic patients with a mutation in the mitochondrial gene in Japan. J Clin Endocrinol Metab 1994;79(3):768-71.

9. Kishimoto $M$, Hashiramoto $M$, Araki S, Ishida $Y$, Kazumi T, Kanda $F$, et al. Diabetes mellitus carrying a mutation in the mitochondrial tRNA LEU(UUR) gene. Diabetes 1995;38:193200.

10. Okubo K, Yamano A, Nagashima M, Mori $Y$, Anzai K, Akehi $Y$ et al. Mitochondrial gene mutations in the tRNA LEU(UUR) region and diabetes: prevalence and clinical phenotypes in Japan. Clin Chem 2001;47:1641-8.

11. Gimeno SGA, Ferreira SRG, Franco LJ, Hirai AT, Matsumura LK, Moises RCS; for the Japanese Brazilian Study Group. Prevalence and 7-year incidence of type 2 diabetes mellitus in a Japanese Brazilian population: an alarming public health problem. Diabetologia 2002;45:1635-8.

12. Alberti KGMN, Zimmet PZ; for World Health Organization. Definition, diagnosis and classification of diabetes mellitus and its complication. Report of WHO consultation. WHO: Geneva, 1999.

13. Massin $P$, Virally-Monod M, Vialettes B, Paques $M$, Gin $H$, Porokhov B; on behalf of the GEDIAM Group. Prevalence of macular pattern dystrophy in maternally inherited diabetes and deafness. Ophthalmology 1999;106:1821-7

14. Service FJ, Rizza RA, Zimmerman RB, Dyck PJ, O'Brien PC Melton LJ. The classification of diabetes by clinical and $\mathrm{C}$ peptide criteria. A prospective population-based study. Diabetes Care 1997;20(2):198-201.

15. Salles JE, Moises VA, Almeida DR, Chacra AR, Moises RS. Myocardial dysfunction in mitochondrial diabetes treated with coenzyme Q10. Diabetes Res Clin Pract 2006;72(1):100-3.

16. Guillausseau PJ, Dubois-Laforgue D, Massin P, Laloi-Michelin $M$, Bellanné-Chantelot $C$, Gin $H$, et al. Maternally inherited diabetes and deafness: a multicenter study. Ann Intern Med 2001:134(9 Pt 1):721-8.

17. Macmillan CJ, Lach B, Shoubridge EA. Variable distribution of mutant mitochondrial DNAs (tRNA(Leu[3243])) in tissues of symptomatic relatives with MELAS: the role of mitotic segregation. Neurology 1993;43(8):1586-90.
18. Shiraiwa N, Ishii A, Iwamoto $H$, Mizusawa $H$, Kagawa $Y$, Ohta S. Content of mutant mitochondrial DNA and organ dysfunction in a patient with a MELAS subgroup of mitochondrial encephalomyopathies. J Neurol Sci 1993;120(2):174-9.

19. Chinnery PF, Zwijnenburg PJ, Walker M, Howell N, Taylor RW Turnbull DM, et al. Nonrandom tissue distribution of mutant mtDNA. Am J Med Genetics 1999;27:85(5):498-501.

20. Suzuky Y, Goto Y, Taniyama M, Nonoka I, Atsumi Y, Matsuoka $\mathrm{K}$, et al. Muscle histopathology in diabetes mellitus associated with mitochondrial tRNA(Leu(UUR)) mutation at position 3243. J Neurol Sci 1997;145(1):49-53.

21. Liu VWS, Zhang $C$, Linnane AW, Nagley $P$. Quantitative allelespecific PCR: demonstration of age-associated accumulation in human tissues of the A-G mutation at nucleotide 3243 in mitochondrial DNA. Hum Mutat 1997;9:265-71.

22. Crispim D, Tschiedel B, Souto KEP, Roisenberg I. Prevalence of three mitochondrial DNA mutations in type 2 diabetic patients from southern Brazil. Clin Endocrinol 2002;57:141-2.

23. Newkirk JE, Taylor RW, Howel N, Bindoff LA, Chinnery PF, Alberti KG, et al. Maternally inherited diabetes and deafness: prevalence in a hospital diabetic population. Diab Med 1997; 14(6):457-60.

24. Saker PJ, Hattersley AT, Barrow B, Hammersley MS, Horton $\mathrm{V}$, Gillmer MD, et al. UKPDS 21: low prevalence of the mitochondrial transfer RNA gene (tRNA(Leu(UUR))) mutation at position $3243 \mathrm{bp}$ in UK caucasian type 2 diabetic patients. Diab Med 1997;14(1):42-5.

25. Ng MCY, Yeung VTF, Chow CC, Li JKY, Smith PR, Mijovict $\mathrm{CH}$, et al. Mitochondrial DNA A3243G mutation in patients with early- or late-onset type 2 diabetes mellitus in Hong Kong Chinese. Clin Endocrinol 2000;52:557-64.

26. $\mathrm{t}^{\prime}$ Hart LM, Lemks HH, Heine RJ, Stolk RP, Feskens EJ, Jansen JJ, et al. Prevalence of maternally inherited diabetes and deafness in diabetic populations in the Netherlands. Diabetologia 1994;37(11):1169-70.

27. Kadowaki T, Kadowaki H, Mori Y, Tobe K, Sakuda R, Susuki $Y$, et al. A subtype of diabetes mellitus associated with a mutation of mitochondrial DNA. N Engl J Med 1994;7:330(14):962-8.

28. Ciafaloni E, Ricci E, Servidei S, Shanske S, Silvestri G, Manfredi $G$, et al. Widespread tissue distribution of a tRNALeu(UUR) mutation in the mitochondrial DNA of a patient with MELAS syndrome. Neurology 1991;41(10):1663-4.

29. Maassen JA. Mitochondrial diabetes: pathophysiology, clinical presentation, and genetic analysis. Am J Med Genet 2002;115:66-70.

30. Narbonne H, Perucca-Lostanlen D, Desnuelle C, Vialettes B, Saunières A, Paquis- Flucklinger V. Searching for A3243G mitochondrial mutation in buccal mucosa in order to improve the screening of patients with mitochondrial diabetes. Eur $\mathbf{J}$ Endocrinol 2001;145:541-2.

31. Manssen JA, Biberoglu S, t'Hart LM, Bakker E, de Knijff P. A case of de novo A3243G mutation in mitochondrial DNA in a patient with diabetes and deafness. Arch Physiol Biochem 2002;110:186-8.

32. Ko CH, Law CW, Tse PWT, Kong CK, Chan AKH, Wong LJC. de novo mutation in the mitochondrial tRNA LEU(UUR) gene (A3243G) with rapid segregation resulting in MELAS in the offspring. J Paediatr Child Health 2001;37:87-90.

33. Yamamoto $M$. Did de novo MELAS common mitochondrial DNA point mutation (mtDNA 3243, A $\rightarrow$ G transition) occur in the mother of a proband of a Japanese MELAS pedigree? $\mathbf{J}$ Neurol Sci 1996;135(1):81-4.

Endereço para correspondência:

Regina S. Moisés

Rua Botucatu 740, $2^{\circ}$ andar

04034-970 São Paulo, SP

Fax: (1 1) 5579-6636

E-mail: rmoises@endocrino.epm.br 